\title{
Autonomía universitaria e integración de América Latina: el papel de la UDUAL
}

\author{
José TADEU JORGE
}

Ex rector de la Universidad Estatal de Campinas y ex presidente de UDUAL

La realización del primer Congreso de Universidades Latinoamericanas, en septiembre de 1949, en Guatemala (por el simple hecho de llevarse a cabo), ya podría ser llamado un avance considerable para las relaciones entre las universidades, y para la creación de referencias que apuntaran hacia el desarrollo de las instituciones existentes. Convocada por la Universidad de San Carlos, la iniciativa del Instituto Americano de Relaciones e Investigaciones Universitarias de Santiago de Chile, reunió 24 universidades de diferentes países de América Latina. En su discurso de bienvenida, al referirse a la ausencia de representantes de las universidades que no pudieron comparecer al evento, el doctor Carlos Martínez Durán, con palabras muy adecuadas, dijo: "Para las universidades ausentes, la puerta del entendimiento comprensivo queda abierta, franca y leal".

El Congreso, entre tanto, fue mucho más allá. Estableció la creación y las bases constitutivas de la Unión de Universidades de América Latina y el Caribe, que adoptaría posteriormente las siglas UDUAL. El texto aprobado trazaba las finalidades del organismo internacional, entre ellas, la base más fundamental: "propender a la implementación de los principios universitarios de libertad en la investigación, libertad de cátedra y de plena autonomía para todas las Universidades Latinoamericanas". Tal objetivo mostraba total sintonía con los principios rectores del evento, también expresados en el discurso de bienvenida para los participantes: "es imprescindible un ambiente político favorable, un clima de alta y hermosa libertad. En el caso especial de la Universidad, ésta debe gozar de autonomía plena, formal y patrimonial".

Los ideales de integración de América Latina, así como el papel social de las universidades, también fueron establecidos de manera clara y propositiva en los objetivos de la nueva entidad asociativa: "contribuir a la realización, en el plano internacional, de los ideales de unidad de la América Latina y en el orden nacional de los postulados de organización democrática, de respeto a la dignidad humana y de justicia social".

Tenemos todavía un último punto a destacar entre los objetivos: "propender a la orientación, coordinación y, en lo posible, a la unificación básica de la organización académica y administrativa de las Universidades de Latinoamérica, 
sin perjudicar la capacidad de conservar e incluso acentuar las diferencias propias del medio en que actúan”.

Para entender la disposición y los propósitos que hicieron que naciera la UDUAL, es necesario revisar las palabras del doctor. Carlos Martínez Durán en el discurso de apertura de los trabajos del primer Congreso de Universidades de Latinoamericanas: "nada despierta tanto la simpatía y el afecto, fortifica la comprensión y genera alegrías, como trabajar juntos para crear y para superarse. Así este congreso es el punto de partida para nuevas y renovadas etapas en la unidad espiritual de América".

A pesar de que los estatutos actuales de la UDUAL tengan otra redacción, sean más comprensivos y mejor adaptados al mundo de hoy, las concepciones originales permanecen fundamentalmente iguales.

\section{¿Y 70 años después, cómo estamos?}

Sabiendo que no es posible responder a tal pregunta analizando todos los aspectos existentes, abordaremos concisamente tres temas que nos parecen esenciales al día de hoy: la autonomía, la producción de conocimiento para beneficio de la sociedad y la integración académica.

Y en el marco conmemorativo del 70 aniversario de una institución, se vuelve oportuno analizar otra cuestión: ¿̨hacia dónde debemos ir?

\section{Autonomía}

Algunos meses antes de la creación efectiva de la UDUAL, en enero de 1949, en el documento titulado Proposición sobre la autonomía, Luis Alberto Sánchez aborda el tema resaltando dos aspectos: las relaciones de las universidades con el Estado y el desarrollo de las propias universidades. En su análisis destaca la diversidad de situaciones existentes en los países latinoamericanos, teniendo algunas universidades plenamente autónomas al lado de otras sin ninguna autonomía. Entre esos extremos, hay varias universidades con distintos grados de libertad a las que denominó semiautónomas. Relaciona, de cierta forma, la existencia de universidades autónomas con la democracia vigente en esos países, lo que es una condición importante para establecer la debida sintonía de las instituciones con relación a las necesidades sociales y de desarrollo. Conceptualmente defendió un modelo de autonomía que englobaba las actividades pedagógicas, financieras, administrativas y doctrinales. Concluyó proponiendo que el Congreso de Universidades Hispanoamericanas aprobara una resolución, cuyo punto más relevante indicaba el establecimiento constitucional de la autonomía universitaria.

A pesar de que el concepto, el alcance y los límites de la autonomía universitaria se hayan discutido hace siglos, es cierto que el siglo Xx marcaría la tendencia para dar formato legal al tema, especialmente en el sentido de adoptarlo como un principio establecido en las leyes supremas de cada país. Valadés (2014, p. 27, 28 y 36) analiza este período enfocándose especialmente en los países de América Latina y demuestra claramente el crecimiento del número de países en los que la constitución incorporó dispositivos que garantizaron la autonomía de las universidades. Hace 
referencia a 17 países de la región que incluyen en sus constituciones los principios de autonomía universitaria, algunos desde tener la simple garantía del principio, hasta otros con detalles más elaborados sobre compromisos y lineamientos. Al analizar la regulación constitucional mexicana y el reporte histórico de adaptación de las instituciones involucradas, se puede constatar que ciertamente puede ser extrapolada para todos los países latinoamericanos y que merece ser recordada y destacada:

La adaptación de las normas relativas a la autonomía universitaria no ha sido fácil. Las comunidades académicas han tenido que aprender a distinguir entre las reglas de las comunidades políticas y las que rigen a las comunidades académicas. Los principios agonistas de competencia y de lucha de la sociedad política no son los propios de un espacio donde se utiliza el diálogo e incluso el debate para construir el saber y no para disputar el poder.

A pesar de todas las dificultades prácticas y de operación encontradas en los procesos de autonomía, es un hecho indiscutible que los avances registrados son muy significativos. Y no es difícil demostrar que universidades realmente autónomas contribuyen de manera más efectiva y decisiva para mejorar las condiciones sociales de las personas, y para producir conocimientos que generen mejor calidad de vida y propicien mayor libertad a cada individuo.

Al asociar enseñanza, investigación y extensión, las universidades vuelven viable la posibilidad de que existan empleos más calificados y con ingresos más significativos para los profesionistas graduados, generan conocimientos que son convertidos en productos y servicios más eficientes y eficaces, y están siempre en sintonía con las necesidades sociales, desde las más inmediatas, hasta aquellas que todavía demandan mejorías.

La conclusión de Cantard es muy oportuna (2014, p. 75): "Las políticas de investigación orientadas a la mejora de la calidad de vida social, permiten generar herramientas científicas y tecnológicas de mayor incidencia en el bienestar de la población, en el fortalecimiento de la democracia, en la reafirmación de las igualdades y en concretar un desarrollo sustentable".

La creación de la UDUAL fue lo que impulsó de manera más organizada la demanda por la autonomía universitaria en América Latina en el siglo pasado. Es verdad que existían antes algunas universidades que ya se regían por este principio, pero las acciones establecidas a partir de entonces permitieron divulgar estas experiencias y darles mayor visibilidad, tanto en el medio académico, como principalmente fuera de él, en especial para los gobiernos y la opinión pública.

El papel incorporado desempeñado por la UDUAL fue decisivo para difundir la idea y los argumentos que podrían ser aportados para defender el principio y aclarar las ventajas de la efectiva implementación de la autonomía universitaria.

A lo largo del tiempo los hechos demuestran la fidelidad de la UDUAL a sus orígenes, con acciones concretas en defensa de la autonomía universitaria, en sus textos, eventos, reuniones y publicaciones. Eso fue y continúa siendo absolutamente necesario, ya que las motivaciones ideológicas 
y mercadológicas aparecen constantemente como amenazas a ese pilar de sustento de las universidades. Debido a contextos así, en septiembre de 2012, la Declaración de Guadalajara sobre Autonomía Universitaria, nuevamente reafirmó sus convicciones al respecto de la autonomía como principio indispensable para las universidades. Aun cuando han pasado casi siete años, nunca está de más recordar su contenido, en nombre de la eterna vigencia que ese principio merece:

Nos pronunciamos por continuar la defensa de la autonomía universitaria, valor que está siendo amenazado no sólo en México, sino a lo largo y ancho de América Latina y el Caribe. En este sentido, fortaleceremos el Observatorio de la Autonomía Universitaria concebido como un espacio para compartir visiones y experiencias sobre este asunto en la región latinoamericana y del Caribe. Por otra parte, asumimos la necesidad de redefinir su concepto para adecuarlo a las circunstancias del presente. Hoy, autonomía universitaria significa: a) El fortalecimiento de la independencia, la legitimidad y la eficacia de los órganos colegiados que gobiernan la vida interna de las universidades; b) El reconocimiento de prerrogativas presupuestarias, por parte de los gobiernos nacionales, que permitan la planeación universitaria a mediano plazo, la certidumbre laboral y la plena vigencia de los derechos laborales de los profesores y empleados universitarios; c) El reconocimiento de las libertades de cátedra, investigación y creatividad humanística de los universitarios; d) El garantizar el financiamiento público de la educación superior como un derecho constitucional que corresponde observar más allá de la aplicación de criterios político-gubernamentales de carácter interesado. En otras palabras, la exigencia de rendición de cuentas a la universidad no debe utilizarse como pretexto para coartar la autonomía universitaria de ninguna manera, exigencia que no va en menoscabo de nuestro compromiso con la calidad en el ejercicio de nuestras funciones; e) Contar con espacios seguros que garanticen la libertad de la comunidad universitaria, en el contexto de los escenarios de inseguridad que actualmente se presentan en México (UDUAL, 2014, p. 22 y 23).

\section{Producción de conocimiento para beneficio de la sociedad}

Dentro de las finalidades de la UDUAL se encuentra fomentar las actividades que permitan integrar licenciaturas, posgrados, investigación y acciones que generen la transferencia y difusión del conocimiento producido. Tales objetivos, sintonizados con el papel social definido para las universidades, en especial aquellas que se sustentan con recursos públicos, resultan estímulos fundamentales para que esas instituciones busquen contribuir con innovaciones que puedan equiparar adecuadamente los grandes problemas que vive la sociedad.

En lo que se dice respecto a América Latina, la importancia del trabajo realizado por las universidades públicas es particularmente decisivo, ya que los otros agentes de la innovación, las empresas y el sector privado, poco invierte o actúan. En un escenario como ese, la existencia de arti- 
culaciones optimiza los esfuerzos y genera resultados más adecuados. Las redes universitarias propician oportunidades de acción conjunta, con elevada sinergia y mayor relación costo-beneficio.

La heterogeneidad latinoamericana se demuestra en toda su plenitud cuando el tema analizado es el sistema de ciencias y tecnología existente en cada país de la región. El aspecto más fundamental, evidentemente, es el financiamiento. En promedio, los países de la región invierten en sus sistemas entre $0.7 \%$ y $0.8 \%$ del PIB anualmente. A pesar de que esas inversiones han estado creciendo a lo largo de los años, todavía están muy distantes de aquellas existentes en países de Europa, China, Canadá, Estados Unidos y todavía más lejanas de aquellos que más invierten en este criterio, como Corea del Sur, Finlandia e Israel. Hay enormes diferencias entre los países de América latina. En cuanto Brasil aplica alrededor del 1.2\% del pib, el que le sigue es Argentina con entre 0.6-0.7\%; Costa Rica, México, Chile Y Uruguay se encuentran aplicando entre el $0.4-0.5 \%$, y todos los demás países están por debajo de estos parámetros. En otro análisis, Brasil, México y Argentina representan cerca del $90 \%$ de las inversiones realizadas en ciencia, tecnología e innovación en los países latinoamericanos.

Como consecuencia, el número de artículos científicos publicados mantiene aproximadamente la misma relación, con Brasil, México, Argentina y Chile representando cerca del $90 \%$ del total. A pesar de eso, el impacto de las publicaciones hechas por los países de América Latina es pequeño, significativamente abajo del promedio mundial. Cabe destacar que ese impacto aumenta cuando los artículos poseen coautoría internacional, especialmente cuando las alianzas son con investigadores de los países que más publican. Las colaboraciones internacionales han crecido a lo largo de los últimos años. Cerca del 45\% de las publicaciones de Argentina y de México poseen colaboración internacional, así como el 32\% de las brasileñas. Las principales alianzas de Brasil en las publicaciones de coautoría son con: Estados Unidos, Reino Unido, Francia, España y Alemania. El primer país de América Latina en aparecer en esta lista es Argentina (lugar 11), seguido de Colombia (14), México (15) y Chile (17) (Clarivate analytics, 2017, p.14, 24 y 26).

La situación ejemplificada por Brasil no difiere mucho de la existente en otros países de la región. Este hecho merece atención especial en cuanto a políticas de estímulo a la integración de América Latina, ya que incrementar esa colaboración puede ser esencial para alcanzar los objetivos propuestos. Además de eso, considerando que América Latina abriga acerca del 5 al 6\% de la población mundial, la inversión en ciencia, tecnología e innovación puede ser considerada insuficiente, ya que se sitúa un poco arriba del 3\% del total invertido a nivel mundial.

Producir conocimiento e innovación depende directamente de la existencia de doctores en grandes cantidades. Eso es consecuencia de un sistema de posgrado bien estructurado, además del financiamiento adecuado. Aquí reside otra deficiencia Latinoamérica.

En cuanto varios países europeos, Australia, Estados Unidos, Nueva Zelanda y Corea del Sur, están graduando a centenas de doctores por cada millón de habitantes al año; Israel, Canadá, Japón y algunos países europeos están en el parámetro de más de una centena; los países de América Lati- 
na mejor posicionados en este criterio, Brasil, México, Chile y Argentina, gradúan (pocas) decenas de doctores por cada millón de habitantes al año (CAPES/MEC, <http://www.clicrbs.com.br/pdf/17554144.pdf>).

Esa cuestión es preocupante, pues la prioridad en los países de la región no parece ser el posgrado, como señala el Informe de 2015 sobre educación superior en Iberoamérica, editado por el Centro Universitario de Desarrollo (CINDA):

Tradicionalmente la matrícula universitaria se ha centrado en el grado, y tal dinámica ha aumentado en los últimos años al calor de la expansión de la cobertura que ha reforzado el modelo de formación profesional, disminuyendo la incidencia relativa de la matrícula de maestrías y doctorados. Así, para el caso particular de América Latina, este nivel, que representó el 3\% del total de la matrícula en 1998, bajó al 2\% entre 1999 y el 2001, y desde el 2002 hasta el 2011 se volvió a reducir para quedar en el 1\%, según la información del Instituto de Estadística de la UNESCO (Barro, 2015, p. 104).

De esta forma, sin financiamiento adecuado y con poca graduación de profesionistas calificados para la producción de conocimiento, América Latina se encuentra frente a enormes desafíos que requieren ser enfrentados para evitar una distancia todavía mayor a la que ahora nos separa de los países más desarrollados.

\section{Integración académica}

Hace 70 años al establecerse los objetivos de la UDUAL, el documento de fundación decía explícitamente que la nueva entidad debería contribuir para "la unificación básica de la organización académica". Evidentemente se mostraba la intención de integrar a la región latinoamericana a través de la educación superior, no sin resaltar los cuidados necesarios para la preservación de las riquezas de cada país con relación a los aspectos culturales históricos.

No es difícil constatar que la propuesta, presentada en 1949, mostraba una teórica visión del futuro, y expresaba mucho más un deseo qué un proyecto que pudiera ser ejecutado basado en esfuerzo y dedicación. La finalidad colocada en los hombros de la UDUAL era instituir, en el ámbito de América Latina, algo como el acuerdo que se materializó en Europa casi 50 años después, con la firma de la Declaración de Bologna, en 1999, y que creó el Espacio Europeo de Educación Superior. Inicialmente constituido por 29 países (hoy cuenta con 48), la implementación fue considerada concluida en 2010, casi 11 años después de que inició. Cabe resaltar que los tratados que viabilizaron la firma de los Ministerios de Educación en el documento de Bologna comenzaron por lo menos una década antes.

En América Latina la semilla plantada en el documento de creación de la UDUAL permaneció latente durante décadas. Entre tanto, la dimensión y el impacto provocado por el proceso de Bologna hicieron renacer el deseo y el tema volvió a la pauta de los medios universitarios latinoamericanos. Solamente en 2008, con la realización de la conferencia regional de educación 
superior en América Latina y el Caribe, en Cartagena de Indias, Colombia, la cuestión volvió a ser tratada en condiciones más objetivas.

La declaración que resultó del citado evento establece:

Es fundamental la construcción de un Espacio de Encuentro Latinoamericano y Caribeño de Educación Superior (ENLACES), el cual debe formar parte de la agenda de los gobiernos y los organismos multilaterales de carácter regional. Ello es básico para alcanzar niveles superiores que apunten a aspectos fundamentales de la integración regional: la profundización de su dimensión cultural; el desarrollo de fortalezas académicas que consoliden las perspectivas regionales ante los más acuciantes problemas mundiales; el aprovechamiento de los recursos humanos para crear sinergias en escala regional; la superación de brechas en la disponibilidad de conocimientos y capacidades profesionales y técnicas; la consideración del saber desde el prisma del bienestar colectivo; y la creación de competencias para la conexión orgánica entre el conocimiento académico, el mundo de la producción, el trabajo y la vida social, con actitud humanista y responsabilidad intelectual (IESALC/UNESCO, 2008).

El plan de acción propuesto específica en una de sus cinco directrices: "promover la integración regional latinoamericana y caribeña y la internacionalización de la educación superior en la región mediante, entre otras iniciativas, la construcción del Espacio de Encuentro de América Latina y el Caribe para la Educación Superior (ENLACES)" (IESALC/UNESCO, 2008).

Después de que se llevará acabo la Conferencia Mundial de Educación Superior, realizada en París en 2009, conforme describe el Documento Base: Líneas de Desarrollo Estratégico, se intensifican las acciones con el objetivo de implantar el ENLACES, que adquiere los primeros contornos concretos de su concepción: a) una plataforma regional de conocimiento e información, y de integración de la enseñanza superior para América Latina y el Caribe; b) un espacio de acción de cooperación solidaria y de articulación entre instituciones; c) una instancia de regulación de procedimientos y sugerencias de normativas; d) un espacio de intercambio de experiencias con base en la movilidad académica y cooperación científica, y que utiliza tanto los elementos de interacción virtual, como real, en pro de la construcción de un espacio de integración de la educación superior capaz de crear vínculos entre los países de América Latina y el Caribe.

El documento base estableció también que el ENLACES fue fundamentado en los siguientes acuerdos centrales: 1 ) constituir el grupo inicial de los ENLACES con un primer conjunto de universidades que estén adheridas a los principios de la cres 2008, de diferentes países de Latinoamérica y el Caribe, y que cumplan con el requisito de ser equivalentes (desarrollen educación de licenciatura y posgrado, investigación y extensión, con calidad), procurando fortalecer la enseñanza superior de carácter solidario con la incorporación de otras universidades e instituciones que cumplan con esos requisitos; 2) que esas universidades establezcan programas académicos para licenciatura, bachillerato, maestría y doctorado con el objetivo de dar los primeros pasos para la creación de un sistema de créditos comunes, que 
posibilite transferencias y reconocimientos; 3) que todo el proceso tendrá que ser coordinado por los autores, o sea, por los rectores de las universidades participantes.

Hoy el ENLACES está legalmente constituido con sus estatutos aprobados, y que definen como finalidades "articular, integrar y representar la educación superior de América Latina y el Caribe”. Los requisitos que deben ser cumplidos para poder afiliarse son: "ser una conferencia nacional de rectores, organización regional de instituciones de educación superior y/o redes e instituciones de educación superior propiamente dichas, que compartan las finalidades, principios y objetivos del ENLACES".

Desde el comienzo de las iniciativas que visualizaban la creación del ENLACES, la UDUAL desempeñó un papel protagónico y en total sintonía con su origen e historia. Teniendo como una de sus finalidades el apoyo a la integración académica de las universidades latinoamericanas, actuó en las articulaciones entre instituciones, organizó reuniones y eventos, produjo informes y textos, y asumió responsabilidades cuando el espacio comenzó funcionar efectivamente.

Dependía de la UDUAL el constituir y coordinar al grupo inicial de universidades que participó en el proyecto piloto, cuyo objetivo fue analizar las disciplinas y los programas de siete licenciaturas (administración, enfermería, ingeniería civil, filosofía, física, química y matemáticas) para verificar la compatibilidad y la posible definición de una metodología para reconocer créditos y títulos en el ámbito de esas instituciones de educación superior. El grupo estaba conformado por las siguientes instituciones: Universidad Estatal de Campinas, Universidad Federal de Minas Gerais, Universidad de La Habana, Pontificia Universidad Católica del Perú, Universidad Nacional de Avellaneda, Universidad Nacional de San Juan y la Universidad de Panamá. Al principio también participó la Universidad Nacional de Colombia. La reunión para dar inicio a los trabajos ocurrió en junio de 2015, en la Universidad de Panamá, y los primeros acuerdos fueron concluidos recientemente.

\section{Los próximos años}

La historia está repleta de episodios en los que la autonomía de las universidades fue cuestionada, puesta a prueba y atacada. El ambiente universitario estimula la competencia, lo contradictorio y el libre debate de las ideas. Ese es el contexto que permite crear condiciones para que se produzca un nuevo conocimiento. El estímulo a la osadía es esencial. Los gobiernos basados en regímenes autoritarios e intolerantes no sobreviven por mucho tiempo si existe libertad de expresión, son rápidamente contrariados por datos y argumentos científicos consistentes y no resisten al debate intelectual promovido constantemente por las universidades. Con el objetivo de mantener el poder actúan intentando silenciar las voces calificadas, atacando al raciocinio lógico y fundamentado.

Recientemente el presidente de la Sociedad Alemana de Amparo a la Investigación, Peter Strohschneider, declaró: "Los regímenes autocráticos temen a la libertad de las ideas y al debate intelectual promovido en las universidades y siempre van a intentar restringir su autonomía" (Inves- 
tigación FAPESP, 2019, p.35). Citó también algunos ejemplos actuales de temas y países en los que la intolerancia de los gobiernos provoca reflejos en autonomía universitaria.

Podemos todavía agregar una alerta siempre actual que fue expresada con mucha claridad y propiedad por Dias Sobrinho:

La autonomía no es una dádiva recibida por la universidad. Antes que eso, es una conquista que necesita ser asegurada permanentemente para la optimización del ejercicio de las funciones públicas y de responsabilidad social de las universidades. Las instituciones necesitan ser merecedoras de la autonomía frente al Estado y a las conexiones de los poderes gubernamentales con el mercado. La defensa de la autonomía es esencial para la sobrevivencia de la universidad pública. Pero sólo el cumplimiento real de las funciones sociales de la universidad podrá asegurar el reconocimiento y el respeto de este principio por parte del Estado (2014, p. 178).

Muchos países consagran el principio de la autonomía universitaria como un dispositivo constitucional, volviéndolo así más sólido y resistente a las objeciones populistas y autoritarias. Entretanto, en muchos otros, apenas los dispositivos menores como leyes y decretos sustentan la vivencia autónoma, y en algunos otros, todavía no hay reglamentos establecidos y se depende totalmente de la voluntad de los gobernantes.

Hay varias situaciones en las que la autonomía está establecida en papel, pero los recursos destinados a las instituciones dependen completamente de la buena voluntad de los gobernantes. No hay autonomía plena sin financiamiento adecuado. La vinculación de los recursos destinados a las universidades, a la recaudación de impuestos, constituye una medida adecuada, estimulante de la autonomía, y representa efectivamente la posibilidad del pleno ejercicio del principio de autonomía.

De esta forma, sea por causa de las constantes amenazas, o para demostrar su contribución a la sociedad, la autonomía universitaria exige un permanente estado de defensa, papel que en la UDUAL debe, y precisa, continuar desempeñando. Al mismo tiempo, debe contribuir para fortalecer con argumentos y demostraciones de sus asociadas, la lucha para hacer constar los textos constitucionales en garantía del principio más fundamental de la existencia de una universidad, así como la especificación de los recursos para el funcionamiento adecuado, vinculados a la recaudación impuestos que paga la sociedad.

En lo que respecta a la producción de conocimiento para beneficio de la sociedad, hay muchos desafíos a enfrentar. Es innegable la necesidad de adecuar el nivel de financiamiento existente, hoy mucho más bajo de lo que sería razonable y esperado para las acciones de investigación e innovación. Una región que acoge cerca del $6 \%$ de la población mundial no puede contribuir únicamente con poco más del 3\% de los recursos mundiales destinados a estas actividades. El mismo raciocinio puede aplicarse a la cuestión de formación de recursos humanos calificados, destinados al desarrollo de las actividades de investigación, innovación y colocación del conocimiento a disposición de la sociedad. La formación de doctores 
y carreras científicas claras y atrayentes son urgentes para los países de América Latina. Puede verse como una cuestión de sobrevivencia, el dejar de graduar únicamente pocas decenas de doctores por millón de habitantes al año, como ocurre en los países de la región, y pasar por lo menos a una centena, es decir, por lo menos duplicar el número de graduados. Esto nos hace referencia a los medios necesarios para alcanzar esos números, que son los cursos de posgrado. Existe la necesidad de implantar programas en áreas emergentes, perfeccionar los perfiles existentes, mejorar la infraestructura y ampliar de manera significativa la capacidad de formación.

No se trata sólo de ampliar la inversión. Existen otras acciones de igual importancia que producen un efecto catalizador en el proceso de formar de doctores. Algunos ejemplos son: a) doctorados conjuntos, impartidos por varias universidades, pudiendo crear áreas de excelencia donde aún no existen, a partir de grupos más consolidados en otras universidades; b) proyectos de investigación conjunta, con características semejantes al punto anterior y la optimización de las inversiones; c) cursos temáticos de posgrado, congregando universidades de diferentes países en temas como la seguridad alimenticia, energía, agua, saneamiento, violencia, tecnología de la información entre otros; d) profesores visitantes, intercambio de profesores para formar grupos de investigación en su área de especialidad en distintos países.

Para que esas iniciativas prosperen se necesita un agente articulador que realice el papel de acercar a las instituciones, proponer iniciativas concretas y buscar agregar socios públicos o privados. Parece evidente la capacidad concentrada en la UDUAL para realizar esta misión: 230 universidades e instituciones de educación superior, localizadas en 22 países de América Latina y el Caribe; cerca de 20 asociaciones y redes universitarias; y 11 organismos de cooperación y estudio. Con la articulación de la UDUAL, esos actores del escenario académico latinoamericano podrán dar el salto de calidad necesario y contribuir para que se alcancen los resultados que coloquen a la región en condiciones de producir ciencia e innovación de una forma más expresiva. En algunas de esas cuestiones la UDUAL ya realizó actividades e iniciativas que precisan ser multiplicadas.

En relación con la integración académica, no hay dudas sobre el camino que se debe seguir. La prioridad absoluta es avanzar con el ENLACES. Es indispensable acelerar su implementación ampliando el número de universidades participantes, y extendiendo la iniciativa a muchos otros cursos. Se ha vuelto urgente definir una metodología que pueda ser empleada de manera general para asegurar el reconocimiento de créditos y títulos entre las universidades de distintos países de América Latina.

La iniciativa más reciente de la Unión Europea nos muestra que estamos muy lejos del nivel que este continente ha alcanzado. En junio de 2019 fue anunciado el resultado de la selección realizada para establecer las primeras 17 universidades europeas. Están dentro de este proyecto 114 instituciones de educación superior de Europa, formando parte las 17 universidades en 24 Estados-miembro de la comunidad europea. El objetivo de esta iniciativa, según la Comisión Europea (2019), "es reunir una nueva generación de europeos creativos, capaces de poner en práctica una cooperación transnacional, transdisciplinaria y en diferentes idiomas, para hacer frente 
a los grandes desafíos sociales y a la escasez de competencias a la que se está enfrentando Europa”. En el comunicado de prensa se define la idea conceptual de esta forma:

Las universidades europeas se convierten en un campus interuniversitario en el que los estudiantes, los doctores, el personal y los investigadores pueden circular sin barreras. Reunir sus conocimientos especializados, plataformas y recursos para la realización de programas o módulos comunes que abarquen varias disciplinas. Estos programas serán muy flexibles y permitirán a los estudiantes personalizar su educación, escogiendo lo que quieren estudiar, dónde y cuándo hacerlo, así como dónde desean obtener su diploma europeo. Las universidades europeas contribuirán también para el desarrollo sustentable de las regiones donde se encuentran, ya que sus estudiantes trabajarán en estrecha colaboración con empresas, autoridades municipales, académicos e investigadores, para encontrar soluciones a los desafíos que enfrentan sus regiones.

Como se puede leer, hay un largo camino por recorrer en la búsqueda del integración académica de los países latinoamericanos. El tema presente en el origen de la UDUAL continúa actual y necesita de avances rápidos.

Que la historia y las iniciativas ya vividas sirvan de estímulo para un arranque definitivo del ENLACES. Una cuestión de esencial relevancia debe de ser recordada siempre: la concepción y coordinación del espacio son atribuciones de las universidades, por lo menos hasta la consolidación del proceso, una vez que es esencial que los conceptos fundamentales de sustentación del proyecto tengan las bases académicas correctas y necesarias, y sean gobernadas por los valores universitarios. 


\section{Bibliografía}

Barro, Senén (coordinador). "La transferencia de I+D, la innovación y el emprendimiento en las universidades”. Educación superior en Iberoamérica. Informe 2015. Santiago de Chile, cindA.

Cantard, Albor (2014). "La autonomía universitaria hoy. Um debate necessário”. In: Villar, Alejandro (compilador), La autonomía universitaria, una mirada latinoamericana. Ciudad de México, UduAL.

Clarivate Analytcs (2017). "Investigaciòn en Brasil, un reporte para CAPEs". Disponible en: <https://www.capes.gov.br/images/stories/download/diversos/17012018-CAPES-InCitesReport-Final.pdf>.

Comisión Europea (2019). Iniciativa de Universidades Europeas”. Disponible en: <https://ec.europa. eu/education/education-in-the-eu/european-education-area/european-universities-initiative_pt> e <https://europa.eu/rapid/press-release_IP-19-3389_pt.htm>.

Dias Sobrinho, José (2014). "Autonomía universitária: bien público y responsabilidad social”. En Villar, Alejandro (compilador), La autonomía universitaria, una mirada latinoamericana. Ciudad de México, udual.

ENLACES (2015). Documento Base. Disponible en: <http://espacioenlaces.org/wp-content/ uploads/2015/12/Documento-base-Lineas-para-el-desarrollo-Estrat\%C3\%A9gico-de-ENLACES..pdf>.

ENLACES (2016) Estatutos. Disponible en: <http://espacioenlaces.org/wp-content/uploads/2018/08/ ESTATUTO-ENLACES-web.pdf>.

ENlaCes (2018) Informe del Comité Ejecutivo Provisional a la Primera Conferencia General Ordinaria. Disponible en: <http://espacioenlaces.org/wp-content/uploads/2018/07/ENLACES-informe-trayectoria.pdf $>$.

IESAlC-unesco (2008). Declaración y Plan de Acción de la Conferencia Regional de Educación Superior en América Latina y el Caribe. Madrid, Ministerio de Ciencia e Innovación / Gobierno de España.

Martínez Durán, C. (1949). Discurso de bienvenida. Disponible en: <https://www.UDUAL.org/ principal/wp-content/uploads/2019/06/Discurso-bienvenida1.pdf >.

Sánchez, Luis Alberto (1949). Proposición sobre autonomia! Disponible en: <https://www.UDUAL. org/principal/wp-content/uploads/2019/06/UDUAL-15.pdf>.

Strohschneider, Peter (2019). "Entrevista". Revista de Investigaciòn FAPESP, año 20, número 280. Disponible en: <https://revistapesquisa.fapesp.br/en/2019/07/04/peter-strohschneider-freedom-to-seek-the-unknown/>.

Unión de Universidades Latinoamericanas (1949). Boletin Informativo, Año I, Número 1.<https:// www.UDUAL.org/principal/wp-content/uploads/2019/06/Documento3.pdf>.

Unión de Universidades de América Latina y el Caribe (2012). "Declaración de Guadalajara sobre Autonomía Universitaria”. En Villar, Alejandro (compilador) (2014), La autonomía universitaria, una mirada latinoamericana. Ciudad de México, uduAL.

Unión de Universidades de América Latina y el Caribe (2014) Estatutos. Disponible en: <https:// www.UDUAL.org/principal/wp-content/uploads/2017/10/Estatutos-UDUAL-2016.pdf>.

Valadés, Diego (2014). "Autonomía y constitución en América Latina”. En Villar, Alejandro (compilador), La autonomía universitaria, una mirada latinoamericana. Ciudad de México, UduAL. 